\title{
CYCLIC ALGEBRAS OF SMALL EXPONENT
}

\author{
J.-P. TIGNOL
}

\begin{abstract}
We prove that every cyclic algebra of exponent $n$ and degree $m n$ over a field containing a primitive $n$th root of unity is similar to a tensor product of at most $m$ symbols of degree $n$.
\end{abstract}

1. Introduction. Let $F$ be a field containing a primitive $n$th root of unity $\omega$. A central simple $F$-algebra of degree $n$ (i.e. of dimension $n^{2}$ ) is called a symbol if it is generated by two elements $x, y$ subject to the relations $x^{n} \in F^{*}, y^{n} \in F^{*}$ and $y x=\omega x y$ (compare [3,§15]). Merkurjev and Suslin [2] have recently proved that every finite-dimensional central simple $F$-algebra of exponent $n$ (i.e. whose similarity class has order $n$ in the Brauer group $\operatorname{Br}(F)$ ) is similar to a tensor product of symbols of degree $n$.

The aim of this note is to give a simple proof of this theorem for cyclic algebras, i.e. for central simple algebras which contain a cyclic extension of the center as a maximal commutative subalgebra.

THEOREM. Let $F$ be a field containing a primitive nth root of unity. Every cyclic $F$-algebra of exponent $n$ and degree $m n$ is similar to a tensor product of at most $m$ symbols of degree $n$.

If $K$ is an extension of a field $F$, we denote by $\operatorname{Br}(K / F)$ the kernel of the natural map from $\operatorname{Br}(F)$ to $\operatorname{Br}(K)$ and by $\operatorname{Br}_{n}(K / F)$ the subgroup of $\operatorname{Br}(K / F)$ which is killed by $n$.

2. Lemma. Let $K / F$ be a cyclic field extension and let $L$ be an intermediate field. Let $n=[K: L]$. Then, the image of the corestriction map

$$
\operatorname{Cor}_{L / F}: \operatorname{Br}(K / L) \rightarrow \operatorname{Br}(K / F)
$$

is $\mathrm{Br}_{n}(K / F)$.

Proof. Let $G$ be the Galois group of $K$ over $F$ and let $\chi$ be a generator of the group $\operatorname{Hom}(G, \mathbf{Q} / \mathbf{Z})$ of characters of $G$. By [6, Corollary 2, p. 211], every element of $\operatorname{Br}(K / F)$ is of the form $(\chi, a)$ for some $a \in F^{*}$. (If we denote by $\sigma$ the generator of $G$ such that $\chi(\sigma)=[K: F]^{-1}(\bmod \mathbf{Z})$, then $(\chi, a)$ is the similarity class of the cyclic algebra $(K, \sigma, a)$, with the notations of $[1$, p. 74].) If $(\chi, a)$ is killed by $n$, then $(n \chi, a)=0$.

Received by the editors December 29, 1982.

1980 Mathematics Subject Classification. Primary 16A16; Secondary 13A20, 12G05.

Key words and phrases. Cyclic algebras, Brauer group of fields.

${ }^{1}$ The author wishes to thank M. Ojanguren for calling [4] to his attention. 
Since the kernel of $n \chi$ is the Galois group of $K$ over $L$, we then have $a=N_{L / F}(t)$ for some $t \in L^{*}$, by [6, Corollary 1, p. 211], whence $(\chi, a)=\operatorname{Cor}_{L / F}\left(\operatorname{Res}_{L / F} \chi, t\right)$, by the "projection formula" (see [6, p. 212 or 7, Proposition 4.3.7]). This proves that the image of $\operatorname{Br}(K / L)$ by the corestriction map contains $\operatorname{Br}_{n}(K / F)$. The converse is clear, since the exponent of $\operatorname{Br}(K / L)$ divides $[K: L]=n$. Q.E.D.

3. Proof of the Theorem. Let $K$ be a cyclic extension of $F$, of rank $m n$. The Lemma shows that every element in $\operatorname{Br}_{n}(K / F)$ is the corestriction of some element in $\operatorname{Br}(K / L)$, where $L$ is the (unique) extension of $F$ of codimension $n$ in $K$. Since $L$ contains a primitive root of unity, every element in $\operatorname{Br}(K / L)$ is the similarity class of a symbol of degree $n$ and, since $[L: F]=m$, the corestriction of any symbol of degree $n$ over $L$ is similar to the tensor product of at most $m$ symbols of degree $n$ over $F$, by a theorem of Rosset and Tate $[4, \S 3$, Corollary 1]. Q.E.D.

4. Remarks. (1) If $n$ is a product of relatively prime integers $n=n_{1} \cdots n_{r}$ then, by [1, Theorem 7.20], every cyclic algebra $A$ of exponent $n$ is isomorphic to a tensor product $A \simeq A_{1} \otimes \cdots \otimes A_{r}$, where $A_{i}$ is a cyclic algebra of exponent $n_{i}$ for $i=1, \ldots, r$. The Theorem above can thus be applied separately to $A_{1}, \ldots, A_{r}$; this yields a better bound for the number of factors in a decomposition of $A$ as a tensor product of symbols (up to similarity). If $n$ is a power of a prime integer, it is not known whether the bound is the best possible. (It is obviously so for $m=1$ or 2.)

(2) For $n=2$, the Theorem above has also been proved by Rowen [5, Theorem $3.7]$, under the extra hypothesis that $F$ contains a primitive $2 m$ th root of unity. His techniques are different and do not yield a bound on the number of symbols.

\section{REFERENCES}

1. A. A. Albert, Structure of algebras, Amer. Math. Soc. Colloq. Publ., vol. 24, Amer. Math. Soc., Providence, R. I., 1939.

2. A. S. Merkurjev and A. A. Suslin, K-cohomology of Severi-Brauer varieties and norm residue homomorphism, preprint, 1981.

3. J. Milnor, Introduction to algebraic K-theory, Ann. of Math. Studies, no. 72, Princeton Univ. Press, Princeton, N. J., 1971.

4. S. Rosset and J. Tate, $A$ reciprocity law for $K_{2}$-traces, preprint, 1982.

5. L. H. Rowen, Central simple algebras with involution viewed through centralizers, J. Algebra 63 (1980), $41-55$.

6. J.-P. Serre, Corps locaux, Hermann, Paris, 1968.

7. E. Weiss, Cohomology of groups, Academic Press, New York, 1969.

Institut de Mathematique, Universite Catholique de Louvain, B-1348 Louvain-la-Neuve, BELGIUM 